\title{
Multiscale Pedestrian Dynamics and Infection Spread Model for Policy Analysis
}

\author{
Sirish Namilae $^{1 *}$, Pierrot Derjany ${ }^{1}$, Dahai Liu ${ }^{1}$ Anuj Mubayi² and Ashok Srinivasan ${ }^{3}$ \\ ${ }^{1}$ Embry-Riddle Aeronautical University \\ Daytona Beach, Florida, USA \\ ${ }^{2}$ Arizona State University \\ Tempe, Arizona, USA \\ ${ }^{3}$ University of West Florida \\ Pensacola, Florida, USA \\ *namilaes@erau.edu
}

\begin{abstract}
In this paper, we present a formulation for a multiscale model combining a social force based pedestrian movement including collision avoidance and a stochastic infection dynamics framework to evaluate the spread of multiple infectious diseases during air travel. We apply the multiscale model to evaluate pedestrian movement strategies that can reduce infection spread during air travel. The results are presented for airport lounge and airplane boarding and deplaning. Use of parallel computing to evaluate the vast parameter space created due to stochasticity and discretionary pedestrian behavior is addressed
\end{abstract}

Keywords: Pedestrian dynamics, Infectious disease spread, Air travel

\section{Introduction}

Public transportation in general and air travel in particular have been identified as leading factors in the spread of infectious diseases; there is direct evidence for air travel related spread of infections such as influenza [1], SARS [2], measles [3] and norovirus [4]. Pedestrian movement within an airport and in airplanes is key to understanding and estimating the casual contacts between passengers and thereby understanding the infectious disease spread.

We have formulated a multiphysics computational model $[5,6]$ that incorporates particle dynamics based pedestrian movement of travelers in transit hubs (e.g. airports), contact analysis and stochastic infectious disease spread, to frame and analyze transportation policies that can mitigate infectious disease spread. The high economic and public perception costs on the transportation sector due to pandemic events necessitates policy that addresses mitigation. We utilize this multiscale model for policy design to develop strategies that mitigate spread.

\section{Model Formulation}

In order to first determine the number of pedestrian-pedestrian and pedestrian-surface contacts, we model the dynamics of mobile pedestrians incorporating interactions with other pedestrians and stationary objects, like walls and chairs, as particles. The motion of pedestrians interacting with other pedestrians and stationary particles evolves through molecular dynamics-based social force formulation [7]. The force $\bar{f}_{i}$ acting on pedestrian $i$ (or particle) can be defined as:

$$
m_{i} \frac{d v_{i}}{d t}=\frac{m_{i}}{\tau}\left(\bar{v}_{o}^{i}(t)-\bar{v}^{i}(t)\right)+\sum_{j \neq i} \bar{f}_{i j}(t)
$$

with pedestrian position at a given time obtained by integration as $r_{i}(t)=\int v_{i} d t$ where $\bar{v}_{o}^{i}(t)$ is the desired velocity of pedestrian and $\bar{v}^{i}(t)$ is the actual velocity, $m_{i}$ is the mass and $\tau$ is the time constant. The momentum generated by a pedestrian's intention results in a self-propulsion force that is balanced by a repulsion force $\bar{f}_{i j}(t)$. 
We also introduce location dependence to the desired velocity in the self-propulsion term as: $\bar{v}_{o}^{i}(t) \cdot \hat{e}_{1}=\left(v_{A}+\gamma_{i} v_{B}\right)\left(1-\delta /\left(\bar{r}_{i} \hat{e}_{1}-\bar{r}_{k} \hat{e}_{1}\right)\right)$. Here $\hat{e}_{1}$ is the direction of desired motion. $v_{A}$ and $\gamma_{i} v_{B}$ are the deterministic and stochastic components of desired velocity, $\delta$ is a distance constant such that at distance $\delta$ between pedestrian $i$ and $k$ the desired velocity of pedestrian $i$ is zero. The $\gamma_{i} v_{B}$ term accounts for walking speed variations due to age group and gender [8]. We also introduce path determination and obstacle avoidance into our model to create a realistic representation of pedestrian motion.

Given the trajectory of pedestrians over time, the number of contacts of passenger $i$ at time $\mathrm{t}$ can be evaluated as: $m_{i, t}=\sum r_{i j} \cdot \lambda_{i j}$, where $\lambda_{i j}=0 \quad$ if $r_{i j}>x$ and $\lambda_{i j}=1 / r_{i j}$ if $r_{i j}<x . r_{i j}$ is the distance between pedestrian $i$ and $j$, and $x$ is a virus specific distance parameter. The probability that the susceptible pedestrian $i$ at time $t$ will become infected is computed by multiplying the number of contacts, $m_{i, t}$, with the respective transmission probability of each contact of pedestrian $i$ at time t. Eventually the number of infected individuals will be counted for each time.

Consider a population of size $N$ consisting of $I(t)$ infected and $S(t)$ susceptibles at time t. A susceptible can become infected when coming into direct contact with an infected. However, the newly infected cannot be infective during the start of the incubation period of the illness. Moreover, the infection spread initiates due to the insertion of $i_{c}^{0}$ infectives initially $\left(t_{0}=0\right)$ at their "c" days of infection, out of "d" incubation days. The probability that an infectious individual in the crowd meets other individuals is $m_{i} / N$. Denote by $p_{c}$ the probability that a contact between a susceptible and an infective results in infection of the susceptible. The number of newly infected by infective $i$ at time t, a discrete variable is a Poisson probability distribution, with mean $m_{i}(t-1) . p_{c}$. $[S i(t-1) / N]$. Therefore, the number of people infected at time $t$ is obtained by:

$$
I(t)=\text { Poisson }\left(\sum_{c=1}^{d} \sum_{i=1}^{i_{c}{ }^{0}} m_{i}(t-1) \cdot p_{c} \cdot\left(S_{i}(t-1) / N\right)\right)
$$

Where $m_{i}$ is the number of contact of susceptibles with the infectious traveller $i$ and $p_{c}$ the infection transmission probability.

\section{Results and Discussion}

Figure 1 shows the evolution of pedestrians at airport gate while (a) boarding, (b) deplaning and (c) at airport security check. The evolution of the particles is based on above formulation. We however perform numerous simulations using parallel algorithms on a supercomputing cluster to account for discretionary events and stochasticity in parameters like pedestrian speed. Aggregated information of pedestrian contacts from these simulations is used for modeling infectious disease spread based on Eq. 2 above. The contacts are defined based on time proximity and time spent. For example, for a contact based infectious disease like Ebola, a contact radius within touching distance $(1 \mathrm{~m})$ is used for the simulations while a larger contact radius of $2.1 \mathrm{~m}$ is used for diseases spread through aerosols like H1N1 influenza. This is based on studies that

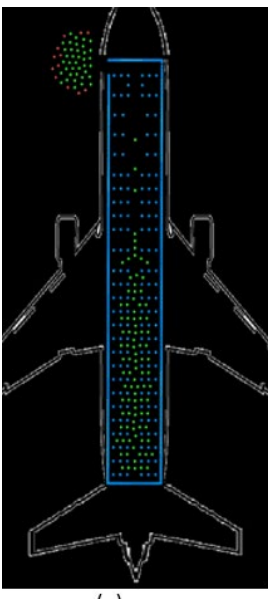

(a)

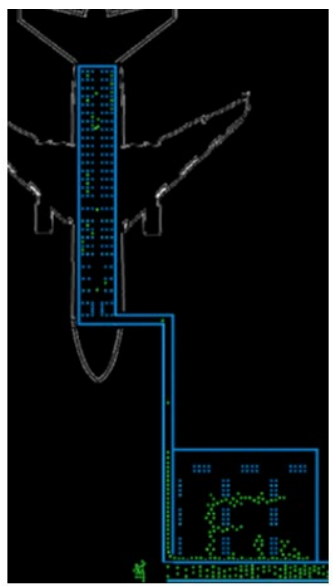

(b)

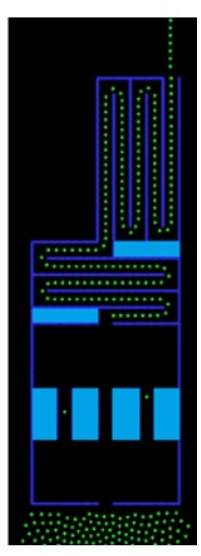

(c)

Fig 1. Simulation snapshots for pedestrian movement for (a) deplaning (b) boarding (c) security check indicate fine particle spread over this distance due to expiratory events like coughing [9]. The probability of infection for different diseases is estimated based on viral shedding e.g. The H1N1 viral RNA level in nasal, oral or ocular shedding is used to construct corresponding probability profiles [10]. 
The combined pedestrian dynamics - infection spread model is then used for parametric analysis of strategies that mitigate infectious disease spread. An example of the policy study is the effect of airplane size and layout on the disease spread. Does transporting same number of people using large or small airplanes help reduce spread? How does this change for diseases transmitted via coarse droplets (e.g. Ebola) Vs fine aerosols (e.g. SARS)? What effect does pedestrian movement during boarding and deplaning have in this conjunction? We find that smaller airplanes are more effective in reducing the number of contacts compared to larger airplanes. Figure 3 provides a comparison between different airplanes and different infectious diseases (Ebola, SARS and H1N1 influenza) for enplaning airplanes of different sizes when one infected

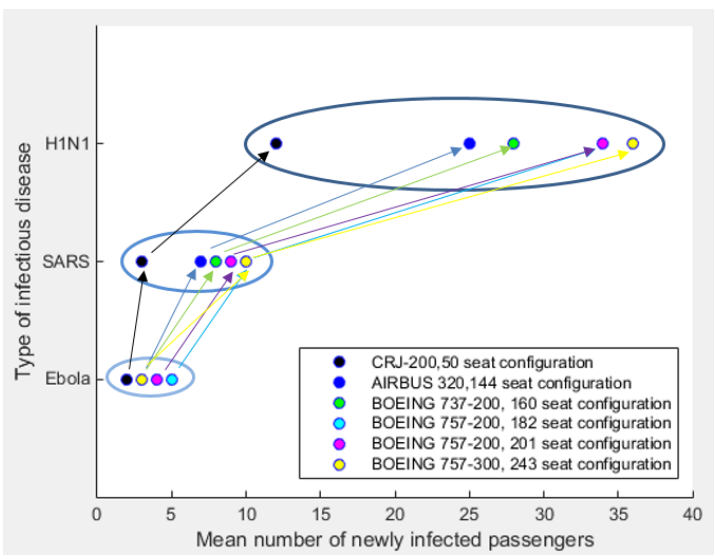

Fig 2. Policy analysis map for airplane size effect on different infectious diseases. passenger in an unknown location is traveling. Such maps can be effective in analysing policies for infection reduction. We have studied similar strategies for queue shape, the size of airport gate and various boarding and deplaning schemes. The final poster will show the variations of infection spread for various strategies.

\section{Conclusion}

A multiscale computational model incorporating pedestrian dynamics and infection spread is presented. The model is used to study infection mitigation strategies for air travel.

\section{Acknowledgements}

Department of Transportation, Center for Advanced Transportation Mobility and National Science Foundation PRAC grant for partially supporting different parts of the research.

\section{References}

[1] M.R. Moser,T.R. Bender, H.S.Margolis, G.R. Noble, A.P. Kendal, and D.G. Ritter, An outbreak of influenza aboard a commercial airliner. American Journal of Epidemiology, 110, (1), 1979, pp.1-6.

[2] S.J. Olsen, H.L.Chang, T.Y. Cheung, A.F.Y. Tang, T.L. Fisk, S.P.L.Ooi, and J. Lando, Transmission of the severe acute respiratory syndrome on aircraft. New England Journal of Medicine, Vol. 349, No.25, 2003, pp. 2416-2422.

[3] K. Nelson, K. Marienau, C. Schembri and S. Redd, 2013. Measles transmission during air travel, United States, December 1, 2008-December 31. Travel Medicine and Infectious Disease, Vol. 11, No.2, 2011, pp. 81-89.

[4] M.A. Widdowson, R. Glass, S. Monroe, R.S. Beard, J.W. Bateman, P. Lurie, and C. Johnson, Probable transmission of norovirus on an airplane. Jama, Vol. 293, No.15, 2005, pp. 1855-1860.

[5] S. Namilae, A. Srinivasan, A. Mubayi, M. Scotch and R. Pahle, Self-propelled pedestrian dynamics model: Application to passenger movement and infection propagation in airplanes, Physica A Vol. 465, 2017, 248-260

[6] S Namilae, P Derjany, A. Mubayi, M. Scotch and A. Srinivasan, Multiscale Model For Infection Dynamics During Air Travel. Physical review E Vol 95, (2017), 052320

[7] D. Helbing, and P. Molnár, Social force model for pedestrian dynamics, Physical Review E, vol. 51, Jan. 1995, pp. 4282-4286.

[8] J. Zębala, P. Ciępka, and A. Reza, Pedestrian acceleration and speeds. Problems of Forensic Sciences, Vol.91, 2012, pp. 227-234.

[9] J.K. Gupta, C.H. Lin and Q Chen, Flow dynamics and characterization of a cough, Indoor Air, vol. 19, 2009, pp. 517-525.

[10] S.C. Paquette.et al Influenza Transmission in the Mother-Infant Dyad Leads to Severe Disease, Mammary Gland Infection, and Pathogenesis by Regulating Host Responses, PLOS Pathogens, vol. 11, Aug. 2015. 